\title{
Relative Brain Size and Its Relation with the Associative Pallium in Birds
}

\author{
Ferran Sayol $^{a}$ Louis Lefebvre ${ }^{a, c}$ Daniel Sol ${ }^{a, b}$ \\ ${ }^{\mathrm{a}} \mathrm{CREAF}$ and ${ }^{\mathrm{b}} \mathrm{CSIC}$, Cerdanyola del Vallès, Spain; ${ }^{\mathrm{c}}$ Department of Biology, McGill University, Montréal, Qué., Canada
}

\section{Key Words}

Encephalization · Brain size - Cognition · Pallium · Mosaic

evolution · Concerted evolution size of the whole brain reflects consistent variation in associative pallium areas and hence is functionally meaningful for comparative analyses.

(c) 2016 S. Karger AG, Basel

\begin{abstract}
Despite growing interest in the evolution of enlarged brains, the biological significance of brain size variation remains controversial. Much of the controversy is over the extent to which brain structures have evolved independently of each other (mosaic evolution) or in a coordinated way (concerted evolution). If larger brains have evolved by the increase of different brain regions in different species, it follows that comparisons of the whole brain might be biologically meaningless. Such an argument has been used to criticize comparative attempts to explain the existing variation in wholebrain size among species. Here, we show that pallium areas associated with domain-general cognition represent a large fraction of the entire brain, are disproportionally larger in large-brained birds and accurately predict variation in the whole brain when allometric effects are appropriately accounted for. While this does not question the importance of mosaic evolution, it suggests that examining specialized, small areas of the brain is not very helpful for understanding why some birds have evolved such large brains. Instead, the
\end{abstract}

\section{Introduction}

The phylogenetic-based comparative approach has become a major tool in investigating the evolution of the vertebrate neural architecture. Much of past effort has been devoted to assessing whether the existing variation in brain size among species predicts differences in cognitively demanding behaviours. This has yielded ample evidence that larger brains are associated with enhanced domain-general cognition [Lefebvre et al., 1997; Reader and Laland, 2002; Reader et al., 2011; Benson-Amram et al., 2016] and function to facilitate behavioural adjustments to socio-environmental changes [Reader and Laland, 2002; Sol et al., 2005, 2007; Sol, 2009; Schuck-Paim et al., 2008]. Despite the progress, the biological significance of brain size variation across species is not exempt from criticism [Healy and Rowe, 2007]. A main argument has been that, because brains are divided into functionally distinct areas, the analyses should focus on the areas

\section{KARGER}

E-Mail karger@karger.com

www.karger.com/bbe (c) 2016 S. Karger AG, Basel

0006-8977/16/0872-0069\$39.50/0
Ferran Sayol

CREAF

Campus UAB, Edifici C

ES-08193 Cerdanyola del Vallès, Catalonia (Spain)

E-Mail f.sayol@ creaf.uab.cat 
Table 1. Encephalization metrics used in the comparative literature on birds

\begin{tabular}{|c|c|}
\hline Metric & References \\
\hline \multicolumn{2}{|l|}{ Frequently used } \\
\hline Log brain mass & Lefebvre and Sol, 2008; Shultz and Dunbar, 2010 \\
\hline Res log (brain) log (body) & Isler and van Schaik, 2006; Franklin et al., 2014 \\
\hline Res log (tel) $\log$ (body) & Nicolakakis and Lefebvre, 2000; Lefebvre and Sol, 2008; Iwaniuk and Wylie, 2006 \\
\hline Res log (tel) log (rest of brain) & Iwaniuk and Wylie, 2006 \\
\hline Volume tel/rest of brain & Shultz and Dunbar, 2010 \\
\hline Log region & Lefebvre and Sol, 2008 \\
\hline Res log (region) $\log$ (body) & Timmermans et al., 2000; Mehlhorn et al., 2010 \\
\hline Res log (region) log (body) log (other regions) & Iwaniuk et al., 2004 \\
\hline Res log (region) log (tel) & Fuchs et al., 2014 \\
\hline Res log (region) log rest of brain) & Iwaniuk and Wylie, 2006; Gutierrez-Ibanez et al., 2014 \\
\hline Martin EQ & Lefebvre and Sol, 2008 \\
\hline Head volume & Møller et al., 2010 \\
\hline Shape based on absolute values & Kawabe et al., 2013 \\
\hline Shape based on regressions against body size & Kawabe et al., 2013 \\
\hline Telencephalon/brainstem of Galliformes & Lefebvre et al., 1997; Zorina and Obozova, 2012 \\
\hline Log tel/brainstem of Galliformes & Lefebvre et al., 1998 \\
\hline Skull height & Winkler et al., 2004 \\
\hline
\end{tabular}

Res = Residual; tel = telencephalon; region = varies according to study (e.g. mesopallium, nidopallium, hyperpallium and visual areas); rest of brain or tel = volume of the brain or telencephalon minus the volume of the region studied.

to which a particular function could be ascribed [Healy and Rowe, 2007].

In fact, the validity of the above criticism depends on the classic, unresolved debate over the extent to which brain areas evolve independently of each other in a mosaic fashion [Barton and Harvey, 2000; Iwaniuk and Hurd, 2005; Barrett and Kurzban, 2006] or in a concerted way as a result of conserved developmental programs [Charvet et al., 2011; Anderson and Finlay, 2013]. If information processing in the brain is massively modular [Barrett and Kurzban, 2006], then larger brains can evolve by the increase of different brain regions in different species, making comparisons of whole-brain size biologically meaningless [Harvey and Krebs, 1990; Healy and Rowe, 2007]. However, if only some areas evolve in a concerted way, but together occupy a large part of the brain, then a disproportionate increase in these brain areas would be reflected in a larger brain regardless of the fact that smaller, more specialized, brain regions might evolve independently. This could be the case of brain areas like the avian mesopallium and nidopallium (which together form the associative pal- lium) and the mammalian isocortex [Rehkämper et al., 1991]. If the most important part of whole-brain size variation is driven by these large, concertedly evolving areas, then focusing on the whole brain in comparative studies would be a good proxy for variation in these areas. Comparative evidence suggests that taxonomic variation in the size of the primate isocortex and the avian associative pallium is associated with variation in a suite of correlated, domain-general cognitive abilities [Lefebvre et al., 2004; Reader et al., 2011] that include feeding innovation and tool use [Timmermans et al., 2000; Lefebvre et al., 2002; Reader and Laland, 2002; Mehlhorn et al., 2010]. Enhanced demands on domain-general cognition could thus be reflected in an enlarged cortex and associative pallium, as well as an enlarged brain.

The debate over models of brain size evolution has not yet been settled in part due to disagreements on how brain size should best be quantified. In primates, as many as 26 different metrics have been used in large-scale studies exploring ecological, life history and cognitive correlates of encephalization [reviewed in Lefebvre, 2012]. The 
comparative literature on birds is similarly based on a variety of metrics, which go from residuals to fractions and proportions of the whole or of parts of the brain (table 1). The different ways in which the data are combined in the analyses adds additional uncertainties about what the size of the whole brain really means [Healy and Rowe, 2007].

In this paper, we use the most complete dataset on avian brain regions currently available [Iwaniuk and Hurd, 2005] to ask what the variation in brain size really means in terms of underlying structures. We use phylogenetically controlled analyses based on the current Bird Tree project [Jetz et al., 2012] to examine inter-relationships between brain size, body size and the volume of 6 major brain parts and to assess the validity of several data transformation metrics used to control for allometry. We predict that a bigger brain should mainly correspond to an increase in associative pallium, and hence that variation in these areas would strongly predict variation in the whole brain when using appropriate methods to remove allometric effects.

\section{Methods}

Data Sources and Phylogenetic Hypotheses

Data on the whole brain and on the volume of 6 brain parts were taken from Iwaniuk and Hurd [2005]. Three regions part of the telencephalon: the nidopallium, which also includes all of the nidopallial subregions [but see Iwaniuk and Hurd, 2005, for more details], the mesopallium and the hyperpallium. Three other nontelencephalic regions include the cerebellum, the diencephalon and the brainstem - which is the sum of the mesencephalon and the myelencephalon. The 6 areas together form between 70 and $87 \%$ of the avian brain volume. Body mass data $(\mathrm{g})$ were obtained from Dunning [2007]. The phylogenetic hypotheses we used were taken from the Bird Tree project [Jetz et al., 2012], where randomly sampled trees were taken from 2 different backbones coming from independent studies [Hackett et al., 2008; Ericson, 2012]. We removed one species (Pavo meleagris) from the database of Iwaniuk and Hurd [2005], as in this set of phylogenetic trees it is considered the same species as Meleagris gallopavo, already present in the database (see online suppl. fig. S1 for an example of one of the phylogenetic hypotheses used; for all online suppl. material, see www.karger.com/doi/10.1159/000444670).

\section{Statistical Analyses}

We first calculated a correlation matrix between the 6 brain areas. We used the 'phyl.vcv' function in R software [R, 2013] with optimization of the parameter lambda using maximum likelihood criteria [Revell, 2012] to account for phylogenetic non-independence of the data. We then compared different ways of removing allometric effects for each brain part, using body mass, volume of the entire brain or volume of a basal part, i.e. the brainstem. For a given brain part, e.g. the nidopallium, we tested the following measures: (1) absolute nidopallium volume; (2) residuals of nidopal- lium volume from a log-log regression against body mass or (3) brainstem volume; (4) nidopallium volume divided by brainstem volume, similar to the executive brain ratio used for primates, and (5) nidopallium volume divided by the volume of the rest of the brain (fraction) or (6) by the volume of the entire brain (proportion). Measures 2 and 3 are thus residuals of log-log regressions and measures 4, 5 and 6 can be calculated using untransformed or log-transformed volumes. We thus had 9 different measures that we compared and tested for potential remaining effects of body size using phylogenetically corrected least-squares regressions (PGLS) with the R package 'caper' [Orme, 2013]. This method, compared to a non-corrected regression, controls for the non-independence of data due to shared ancestry. Contrary to independent contrasts, however, it first determines the strength of the phylogenetic signal in the data (parameter lambda, which varies between 0 and 1 and is calculated using maximum likelihood [Pagel, 1999]) and controls it accordingly, without assuming, as do contrasts, that lambda is 1 . For this purpose, we used a set of 20 phylogenetic trees and calculated means over the 20 models.

For all further analyses, we used residuals only, as other metrics do not eliminate the effect of body mass (see Results). We next analysed the extent to which each brain region is associated with body size using PGLS models with log-transformed variables. To see which brain part best predicts whole-brain variation, we took the residuals of whole-brain volume against body mass and examined their relationship with the residuals of each brain part regressed against body mass. To illustrate these relationships, we plotted positive and negative whole-brain residuals in different shades (black for positive and white for negative) and graphed them against brain part residuals. A brain part that predicts wholebrain size well will yield clearly separated clouds of white and black points; in contrast, a brain part that does not predict whole-brain size well will yield overlapping black and white data points. The extent to which positive and negative whole-brain residuals are well separated in each graph can then be expressed by a histogram illustrating overlaps. We also used a set of PGLS models to determine which allometrically corrected brain part best explains variation in allometrically corrected whole-brain size. A possible problem with the last two analyses is that we are correlating two variables that are residuals from the same predictor (body size), which might lead to some circularity. However, when using brainstem to remove allometry in the brain regions and body size to remove allometry in the whole brain, we obtained exactly the same results in terms of which parts explain most variation in the whole brain.

Finally, we conducted a phylogenetic reconstruction of wholebrain residuals and associative pallium residuals - all corrected for body mass by taking phylogenetic residuals - on a sample tree using the 'contMap' function of the 'phytools' R package [Revell, 2012]. This technique combines data on phylogeny and trait variation between clades to estimate evolutionary increases or decreases in different lineages.

\section{Results}

In terms of absolute size, all brain areas are positively associated with each other in phylogenetically corrected analyses (fig. 1a; online suppl. table S1). Much of this trend 
is due to body size allometry, however, so we next examined the way different transformations of the original data affect the body size confounder. Of all of the metrics we tested, only those based on residuals and the executive brain ratio calculated on log-transformed data completely removed the effects of body size (online suppl. table S2). Analyses based on metrics such as fractions and proportions therefore do not deal exclusively with brain part variation but also include body size.

When allometric effects are taken into account by estimating residuals, some areas show stronger inter-relationships than others, suggesting a combination of concerted and mosaic evolution (fig. $1 \mathrm{~b}$, online suppl. table S3). Concerted evolution is particularly evident for the areas forming the associative part of the telencephalon, notably the nidopallium and the mesopallium $(\mathrm{r}=0.94)$. These two areas show much larger amounts of variation independently of body size than do basal brain areas such as the brainstem (fig. 2; online suppl. table S4). Phylogenetically corrected variation in nidopallium and mesopallium size correctly classifies 95 and $92 \%$, respectively, of the positive and negative residuals of whole-brain size regressed against body size (fig. 2a, b). In contrast, brainstem volume is strongly related to body size and does not discriminate between species with large versus small brain residuals (fig. 2e). As a consequence, brain to body size residuals are better predicted by variation in associative pallium residuals (mesopallium + nidopallium) than by other brain parts (fig. 3), regardless of whether allometry is corrected by body mass (online suppl. table S5) or brainstem volume (online suppl. table S6). In fact, brain size and associative pallium (after corrections for allometric effects) are almost indistinguishable measures of encephalization (fig. 4; PGLS: $\mathrm{R}^{2}=0.91, \mathrm{p}<0.001$ ). Inferring the evolution of avian brains with phylogenetic reconstructions yields virtually identical results with the two metrics (fig. 5), where we can see independent shifts in the increase of both relative brain and associative pallium sizes in crows and parrots and the reduction of these two measures in three practically independent clades (rheids, galliforms and swifts).

\section{Discussion}

Our analyses lead to three main conclusions regarding the evolution of the avian brain. First, all 6 brain parts analysed here tended to increase in a concerted way, a trend that was not simply a consequence of allometry or phylogeny. Second, some areas, notably those belonging

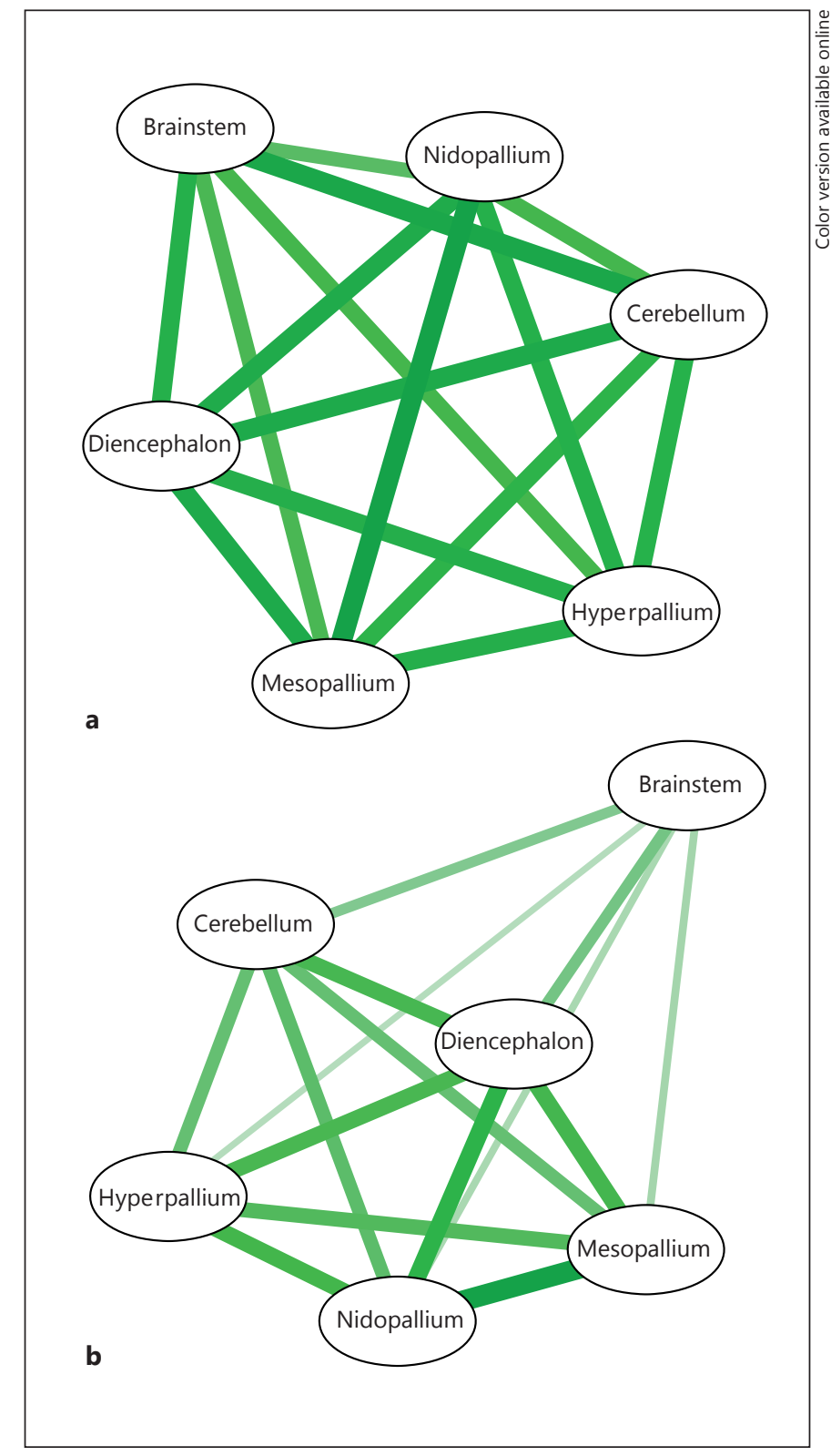

Fig. 1. Phylogenetic correlations between different brain regions, using absolute values (a) or residuals from log-log regressions (b) against body size.
Fig. 2. Log size of the 6 brain parts against log body mass, distinguishing species with positive brain residuals (closed data points) and species with negative brain residuals (open data points). To the right of each plot, we present two histograms, one for each set of dots from the plots (closed and open), corresponding to positive and negative brain residuals. 


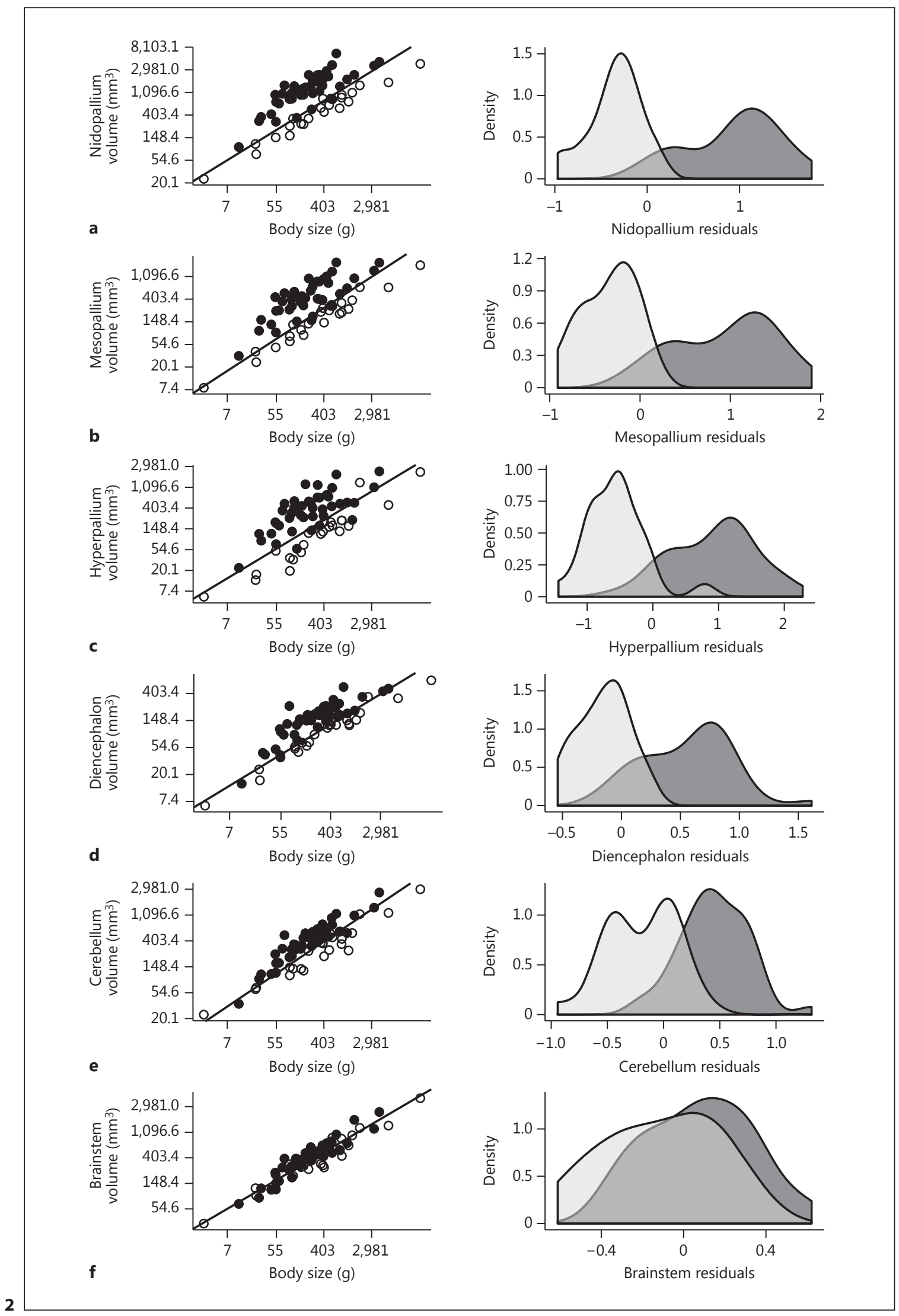




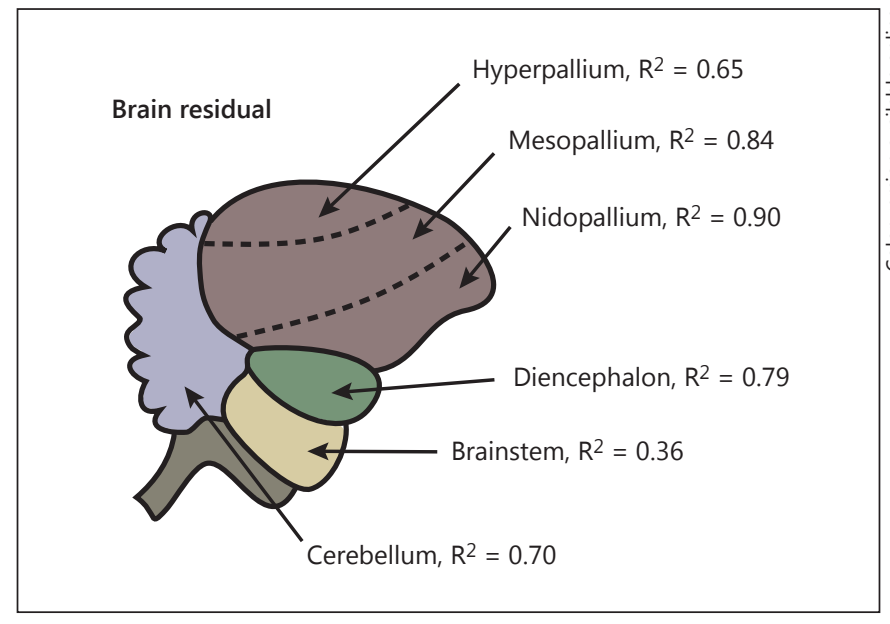

Fig. 3. Relationship between residuals of different brain parts and whole-brain residuals, all regressed against log body mass, with the $\mathrm{R}^{2}$ for PGLS models represented on a schematic avian brain [redrawn based on Nottebohm, 2005].

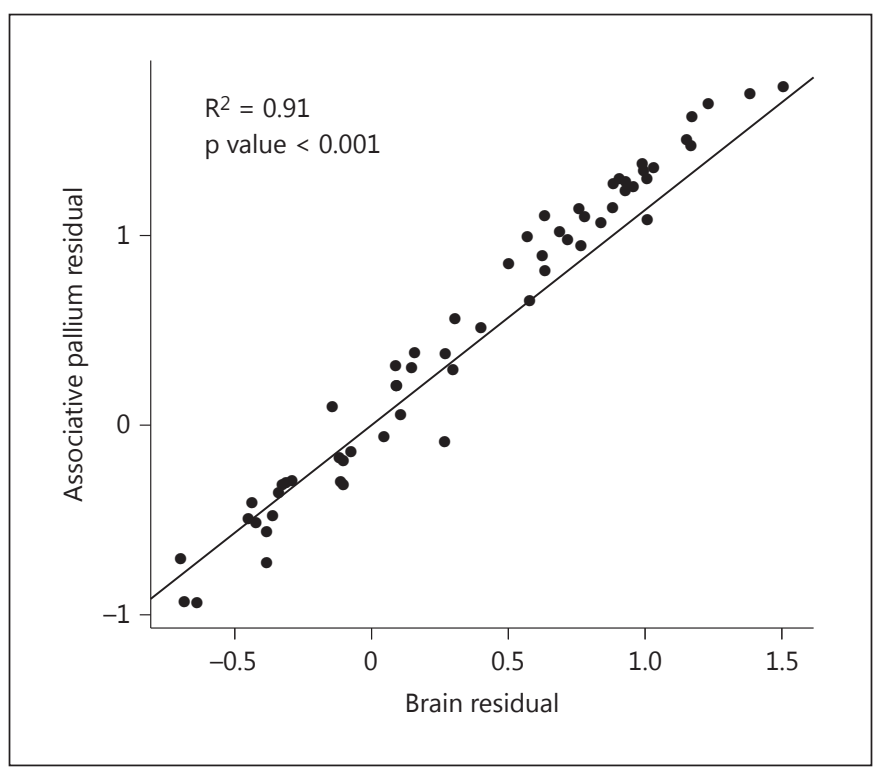

Fig. 4. Residual of whole-brain size against body size plotted against residual of associative pallium size against brainstem size. The data points represent actual species, while the line represents the PGLS model. The slightly lower slope of the regression with respect to the cloud of data points is due to the phylogenetic corrections.

to the associative pallium, evolved in a more concerted way than others. Finally, large brains primarily resulted from a disproportionate increase in these pallial areas. These areas are not only anatomically well delineated (thus minimizing measurement error) but also comprise a large fraction of the brain, in particular the nidopallium. Thus, the same proportional increase of these areas is likely to have a stronger effect on the size of the whole brain than on that of smaller areas, an idea previously proposed by Rehkämper et al. [1991].

The associative pallium areas are known to have key roles in avian cognition. The nidopallium, in particular its caudolateral part, the NCL, is the closest avian equivalent of the mammalian pre-frontal cortex. Several lines of evidence, using different approaches and techniques (connectome [Shanahan et al., 2013], single-unit recording [Rose and Colombo, 2005; Veit and Nieder, 2013; Lengersdorf et al., 2015], receptor architecture [Rose et al., 2010; Herold et al., 2011], temporary inactivation [Helduser and Güntürkün, 2012] and lesions [Mogensen and Divac, 1993]) point to the importance of the NCL in avian executive control. Comparative work also suggests that the nidopallium is the brain area most closely correlated with avian tool use [Lefebvre et al., 2002], while the other part of the associative pallium, i.e. the mesopallium, is most closely correlated with innovation rate [Timmermans, 2000]. The mesopallium is significantly enlarged in the bird with the most sophisticated form of tool use, i.e. the New Caledonian crow (Corvus moneduloides) [Mehlhorn et al., 2010]. The very tight relationship between nidopallium and mesopallium size, once phylogeny and allometry have been removed, further suggests that evolutionary changes in the two structures are strongly linked. Together, the two structures are the closest avian equivalent to the mammalian non-visual cortex. These areas appear to be crucial to domain-general cognitive abilities.

Our results suggest the need for caution in the use of absolute brain size to study the neural basis of cognitive skills, at least in birds. Given that this measure is confounded by body size, traits associated with body size (e.g. range, energetics and prey size) will confound any comparative test of brain size correlates. Using relative measures could be a solution to remove allometric effects, but we found here that dividing brain part volume by the volume of the whole (proportions) or the rest of the brain (fractions), with or without prior log transforms of the volumes, leaves significant body size confounders (table 1). Studies using these metrics [e.g. Clark et al., 2001; Burish et al., 2004] thus contain a hidden confounder that might affect conclusions about evolutionary trends.

In contrast, residual brain size seems to better describe how brains increase due to a disproportionate enlargement of specific, large brain areas. Using residuals completely removes allometric effects on the brain but might pose a problem of interpretation, as it is unclear what a 


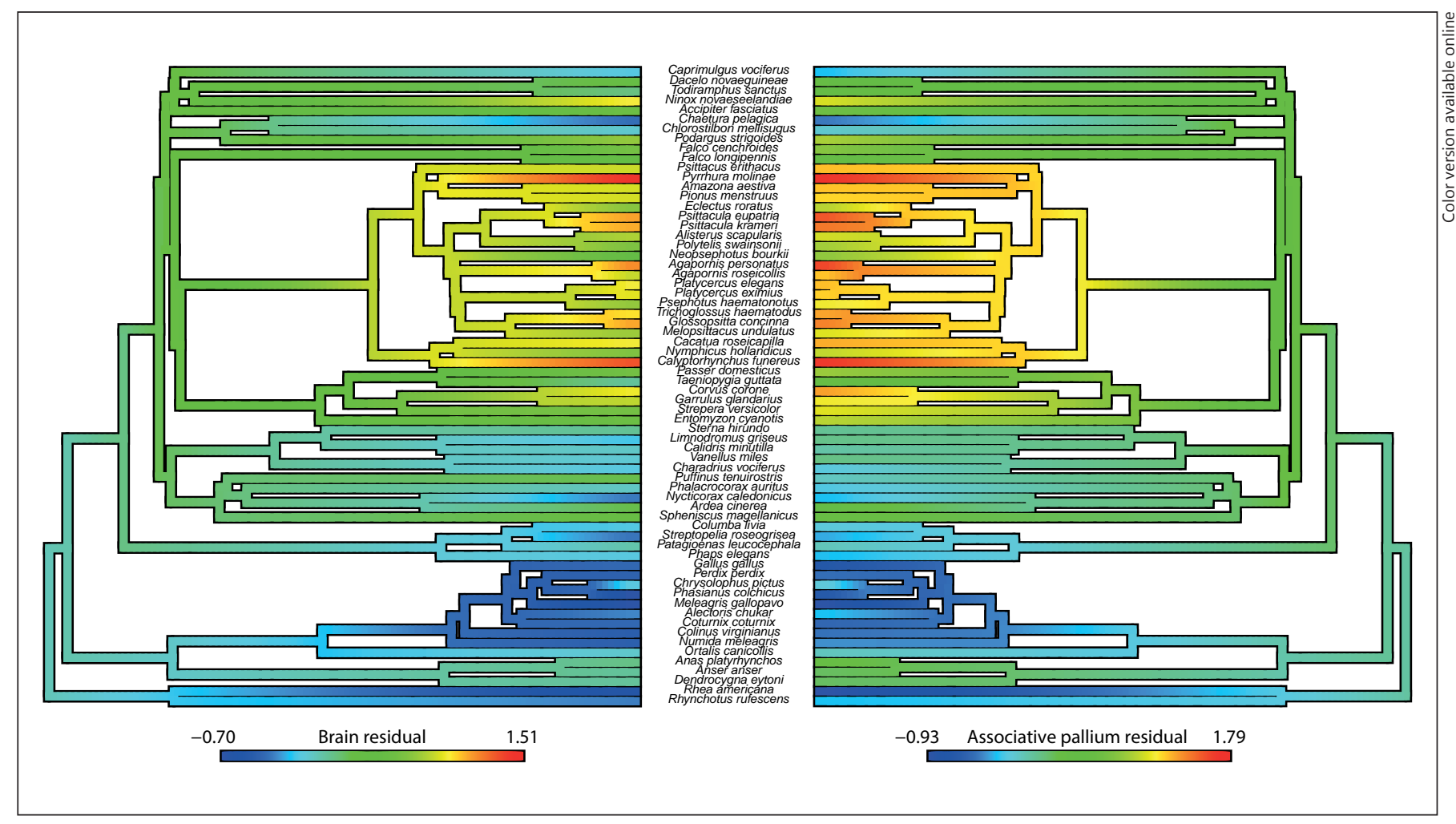

Fig. 5. Phylogenetic reconstruction in a sample phylogenetic hypothesis of birds in our dataset, representing residual brain size evolution and residual associative pallium size evolution.

disproportionately large area means in functional terms. The underlying assumption for existing variation in brain size among species is that any increase in size provides some increase in function. Although this is supported by growing evidence linking residual brain to enhanced cognition [see a revision by Lefebvre and Sol, 2008], why should a disproportionate increase matter at all? Because the brain processes information, and this is done by discrete neurons acting together via neurotransmitters and receptors, the functional significance of volume differences might not be clear. In mammals, different orders have different scaling relationships of neuron numbers to brain area volume [Herculano-Houzel, 2011, 2012]. Similar differences might well characterize bird brains. One can imagine, for example, that a corvid or a parrot mesopallium might have more neurons per cubic millimetre than a quail brainstem. Knowing this would obviously be important, but it would not change correlational trends of the type we report here, or the associations with cognition reported in the literature. We might in fact be underestimating selection on brain areas associated with cognition by focusing on mass or volume rather than neuron numbers if differences in density go in the same direction as differences in classical metrics of encephalization. This also assumes that the number of neurons is the main determinant of information processing capacity, not their connectedness or the density and type of neurotransmitters and receptors. Comparative studies of receptor density and gene expression in brain areas will shed new light on the functional significance of enlarged brains [Goodson et al., 2012].

The finding that enlarged brains have primarily evolved by the concerted increase of certain brain regions does not deny the importance of mosaic evolution. Indeed, the fact that some areas evolve more concertedly than others can be interpreted as a combination of mosaic and concerted evolution. Theoretical work on other biological systems (e.g. metabolic networks [Ravasz et al., 2002]) suggests that modular units are organized into hierarchical clusters, a principle that might reconcile modular and concerted views on the way in which the neural substrate of cognitive abilities operates and evolves. Moreover, mosaic evolution could be more important for small areas specialized in particular be- 
haviours, which have not been evaluated here. A case in point is the network of song nuclei that has been extensively studied in oscines. Nuclei of this type are absent in non-oscines, with the exception of parrots and hummingbirds [Jarvis, 2007], and at least one of them, i.e. the HVC, varies strongly as a result of sexual selection on repertoire size [DeVoogd et al., 1993; Moore et al., 2011]. If there is one clear case of adaptive specialization of brain areas in birds, it is the case of oscine song nuclei, which could evolve independently from other brain regions. However, these findings do not deny that, as our study suggests, the main variation in whole brain size is due to concerted changes in pallial areas, allowing the use of relative brain size as a proxy for relative pallium size in comparative studies.

\section{Acknowledgements}

We are grateful to members of the laboratory of D. Sol for helpful discussions. We also thank two anonymous reviewers for their comments, and José Luis Ordóñez for his help with the figures. This research was supported by funds from the Spanish government (CGL2013-47448-P) to D.S. F.S. was supported by a $\mathrm{PhD}$ fellowship (FI-DGR 2014) from the Catalan government.

\section{References}

Anderson ML, Finlay BL (2013): Allocating structure to function: the strong links between neuroplasticity and natural selection. Front Hum Neurosci 7:918.

Barrett HC, Kurzban R (2006): Modularity in cognition: framing the debate. Psychol Rev 113: 628.

Barton RA, Harvey PH (2000): Mosaic evolution of brain structure in mammals. Nature 405 : 1055-1058.

Benson-Amram S, Dantzer B, Stricker G, Swanson EM, Holekamp KE (2016): Brain size predicts problem-solving ability in mammalian carnivores. Proc Natl Acad Sci USA 113: 2532-2537.

Burish MJ, Kueh HY, Wang SS-H (2004): Brain architecture and social complexity in modern and ancient birds. Brain Behav Evol 63:107124.

Charvet CJ, Striedter GF, Finlay BL (2011): Evodevo and brain scaling: candidate developmental mechanisms for variation and constancy in vertebrate brain evolution. Brain Behav Evol 78:248-257.

Clark DA, Mitra PP, Wang SS (2001): Scalable architecture in mammalian brains. Nature 411 : 189-193.

DeVoogd TJ, Krebs JR, Healy SD, Purvis A (1993): Relations between song repertoire size and the volume of brain nuclei related to song: comparative evolutionary analyses amongst oscine birds. Proc R Soc Lond B Biol Sci 254: 75-82.

Dunning JB (2007): Handbook of Avian Body Masses, ed 2. Boca Raton, CRC Press.

Ericson PGP (2012): Evolution of terrestrial birds in three continents: biogeography and parallel radiations. J Biogeogr 39:813-824.

-Franklin DC, Garnett ST, Luck GW, GutierrezIbanez C, Iwaniuk AN (2014): Relative brain size in Australian birds. Emu 114:160-170.

-Fuchs R, Winkler H (2014): Brain geometry and its relation to migratory behavior in birds. J Adv Neurosci Res 1:1-9.
Goodson JL, Kelly AM, Kingsbury MA (2012): Evolving nonapeptide mechanisms of gregariousness and social diversity in birds. Horm Behav 61:239-250.

Gutiérrez-Ibáñez C, Iwaniuk AN, Moore BA, Fernández-Juricic E, Corfield JR, Krilow JM, Kolominsky J, Wylie DR (2014): Mosaic and concerted evolution in the visual system of birds. PLoS One 9:e90102.

- Hackett SJ, Kimball RT, Reddy S, Bowie RCK, Braun EL, Braun MJ, Yuri T (2008): A phylogenomic study of birds reveals their evolutionary history. Science 320:1763-1768.

- Harvey PH, Krebs JR (1990): Comparing brains. Science 249:140-146

Healy SD, Rowe C (2007): A critique of comparative studies of brain size. Proc R Soc Lond B Biol Sci 274:453-464.

Helduser S, Güntürkün O (2012): Neural substrates for serial reaction time tasks in pigeons. Behav Brain Res 230:132-143.

Herculano-Houzel S (2011): Scaling of brain metabolism with a fixed energy budget per neuron: implications for neuronal activity, plasticity and evolution. PLoS One 6:e17514.

Herculano-Houzel S (2012): The remarkable, yet not extraordinary, human brain as a scaledup primate brain and its associated cost. Proc Natl Acad Sci USA 109:10661-10668.

Herold C, Palomero-Gallagher N, Hellmann B, Kröner S, Theiss C, Güntürkün O, Zilles K (2011): The receptor architecture of the pigeons' nidopallium caudolaterale: an avian analogue to the mammalian prefrontal cortex. Brain Struct Funct 216:239-254.

Isler K, van Schaik C (2006): Costs of encephalization: the energy trade-off hypothesis tested on birds. J Hum Evol 51:228-243.

Iwaniuk AN, Dean KM, Nelson JE (2004): A mosaic pattern characterizes the evolution of the avian brain. Proc Biol Sci 271 Suppl: S148-S151.
Iwaniuk AN, Hurd PL (2005): The evolution of cerebrotypes in birds. Brain Behav Evol 65: 215-230.

Iwaniuk AN, Wylie DRW (2006): The evolution of stereopsis and the Wulst in caprimulgiform birds: a comparative analysis. J Comp Physiol A Neuroethol Sens Neural Behav Physiol 192: 1313-1326.

Jarvis ED (2007): Neural systems for vocal learning in birds and humans: a synopsis. J Ornithol 148:35-44.

-Jetz W, Thomas GH, Joy JB, Hartmann K, Mooers AO (2012): The global diversity of birds in space and time. Nature 491:444-448.

Kawabe S, Shimokawa T, Miki H, Okamoto T, Matsuda S, Itou T, et al (2013): Relationship between brain volume and brain width in mammals and birds. Paleontol Res 17:282293

Lefebvre L (2012): Primate encephalization; in Hofman MA, Falk D: Progress in Brain Research, ed 1. Amsterdam, vol 195.

Lefebvre L, Gaxiola A, Dawson S, Timmermans S, Rosza L, Kabai P (1998): Feeding innovations and forebrain size in Australasian birds. Behaviour 135: 1077-1097.

Lefebvre L, Nicolakakis N, Boire D (2002): Tools and brains in birds. Behaviour 939-973.

Lefebvre L, Reader SM, Sol D (2004): Brains, innovations and evolution in birds and primates. Brain Behav Evol 63:233-246.

Lefebvre L, Sol D (2008): Brains, lifestyles and cognition: are there general trends? Brain Behav Evol 72:135-144.

Lefebvre L, Whittle P, Lascaris E (1997): Feeding innovations and forebrain size in birds. Anim Behav 53:549-560.

-Lengersdorf D, Marks D, Uengoer M, Stüttgen MC, Güntürkün O (2015): Blocking NMDAreceptors in the pigeon's 'prefrontal' caudal nidopallium impairs appetitive extinction learning in a sign-tracking paradigm. Front Behav Neurosci 9:85. 
- Mehlhorn J, Hunt GR, Gray RD, Rehkämper G, Güntürkün O (2010): Tool-making New Caledonian crows have large associative brain areas. Brain Behav Evol 75:63-70.

Mogensen J, Divac I (1993): Behavioural effects of ablation of the pigeon-equivalent of the mammalian prefrontal cortex. Behav Brain Res 55: 101-107.

Møller AP (2010): Brain size, head size and behaviour of a passerine bird. J Evol Biol 23:625635.

- Moore JM, Székely T, Büki J, Devoogd TJ (2011): Motor pathway convergence predicts syllable repertoire size in oscine birds. Proc Natl Acad Sci USA 108:16440-16445.

- Nicolakakis N, Lefebvre L (2000): Forebrain size and innovation rate in European birds: feeding, nesting and confounding variables. Behaviour 137: 1415-1429.

Nottebohm F (2005): The neural basis of birdsong. PLoS Biol 3:0759-0761.

Orme D, Freckleton R, Thomas G, Petzoldt T, Fritz S, Isaac N, Pearse W (2013): Caper: Comparative Analyses of Phylogenetics and Evolution in R. R package (version 0.5.2).

Pagel M (1999): Inferring the historical patterns of biological evolution. Nature 401:877-884.

-Ravasz E, Somera AL, Mongru DA, Oltvai ZN, Barabási A-L (2002): Hierarchical organization of modularity in metabolic networks. Science 297:1551-1555.

RCT (2013): R: A language and environment for statistical computing. R Found Stat Comput. Vienna, Austria.
Reader SM, Hager Y, Laland KN (2011): The evolution of primate general and cultural intelligence. Phil Trans R Soc B Biol Sci 366:10171027.

Reader SM, Laland KN (2002): Social intelligence, innovation, and enhanced brain size in primates. Proc Natl Acad Sci USA 99:4436-4441.

Rehkämper G, Frahm HD, Zilles K (1991): Quantitative development of brain and brain structures in birds (Galliformes and Passeriformes) compared to that in mammals (insectivores and primates) (part 2 of 2). Brain Behav Evol $37: 135-143$.

Revell LJ (2012): phytools: an R package for phylogenetic comparative biology (and other things). Methods Ecol Evol 3:217-223.

Rose J, Colombo M (2005): Neural correlates of executive control in the avian brain. PLoS Biol 3:1139-1146.

Rose J, Schiffer A-M, Dittrich L, Güntürkün O (2010): The role of dopamine in maintenance and distractability of attention in the 'prefrontal cortex' of pigeons. Neuroscience 167: 232-237.

Schuck-Paim C, Alonso WJ, Ottoni EB (2008): Cognition in an ever-changing world: climatic variability is associated with brain size in neotropical parrots. Brain Behav Evol 71: 200-215.

-Shanahan M, Bingman VP, Shimizu T, Wild M, Güntürkün O (2013): Large-scale network organization in the avian forebrain: a connectivity matrix and theoretical analysis. Front Comput Neurosci 7:89.
Shultz S, Dunbar RIM (2010): Social bonds in birds are associated with brain size and contingent on the correlated evolution of life-history and increased parental investment. Biol J Linn Soc 100:111-123.

Sol D (2009): Revisiting the cognitive buffer hypothesis for the evolution of large brains. Biol Lett 5:130-133.

Sol D, Duncan RP, Blackburn TM, Cassey P, Lefebvre L (2005): Big brains, enhanced cognition, and response of birds to novel environments. Proc Natl Acad Sci USA 102:54605465.

Sol D, Székely T, Liker A, Lefebvre L (2007): Bigbrained birds survive better in nature. Proc $\mathrm{R}$ Soc Lond B Biol Sci 274:763-769.

- Timmermans S, Lefebvre L, Boire D, Basu P (2000): Relative size of the hyperstriatum ventrale is the best predictor of feeding innovation rate in birds. Brain Behav Evol 56:196203.

Veit L, Nieder A (2013): Abstract rule neurons in the endbrain support intelligent behaviour in corvid songbirds. Nat Commun 4:2878.

Winkler H, Leisler B, Bernroider G (2004): Ecological constraints on the evolution of avian brains. J Ornithol 145:238-244.

Zorina ZA, Obozova TA (2012): New data on the brain and cognitive abilities of birds. Biol Bull 39:601-617. 